\title{
The Journey to AAPS 2020: a Reflection from Strategic Planning to PharmSci 360
}

\author{
Joseph W. Polli, ${ }^{1,10}$ Christopher R. McCurdy, ${ }^{2}$ Dale Eric Wurster, ${ }^{3}$ Binodh S. DeSilva, ${ }^{4}$ Annette Bak, ${ }^{5}$ \\ Reina Bendayan, ${ }^{6}$ Bernd Meibohm, ${ }^{7}$ Allen C. Templeton, ${ }^{8}$ William Weiser, ${ }^{9}$ and for the AAPS Leadership
}

Published online 3 November 2018

Since its inception in 1986, the American Association of Pharmaceutical Scientists (AAPS) has grown to be one of the premier scientific societies for pharmaceutical scientists in industry, academia, government, and other institutions in North America, with a global outreach to scientists from more than sixty countries around the world. Due to the foresight of its founders, AAPS established itself as a member-driven society that thrives from the scientific expertise and diversity of its membership. The initially-established structure and governance model of AAPS served the organization well for its first three decades. Changes in the competitive landscape of scientific societies, advances in communication technologies, evolving membership expectations, and generational shifts made it necessary to adjust the association's structure and governance model in order to address these challenges and ensure the continued success and longevity of AAPS.

${ }^{1}$ Mechanistic Safety and Drug Disposition, Room 27-3053, GlaxoSmithKline, 709 Swedeland Road, King of Prussia, Pennsylvania 19406, USA.

${ }^{2}$ Department of Medicinal Chemistry, College of Pharmacy, University of Florida, Medical Science Building, P6-33, 1345 Center Drive, PO Box 100485, Gainesville, Florida 32610, USA.

${ }^{3}$ Division of Pharmaceutics and Translational Therapeutics, 308 Pharmacy Building, College of Pharmacy, The University of Iowa, Iowa City, Iowa 52242, USA.

${ }^{4}$ Bristol-Myers Squibb, One Squibb Drive, Mail Stop B105 2437, Building 105, Room 2485, New Brunswick, New Jersey 08901, USA.

${ }^{5}$ Advanced Drug Delivery, Pharmaceutical Sciences, IMED Biotech Unit, AstraZeneca, Gothenburg, Sweden.

${ }^{6}$ Department of Pharmaceutical Sciences Leslie Dan Faculty of Pharmacy, University of Toronto, 144 College Street, Toronto, Ontario M5S 3M2, Canada.

${ }^{7}$ The University of Tennessee Health Science Center, College of Pharmacy, Room 435, 881 Madison Avenue, Memphis, Tennessee 38163, USA.

${ }^{8}$ Merck and Co Inc, RY119-A100, 126 E Grand Ave, Rahway, New Jersey 07065 , USA.

${ }^{9} 3601$ Sweeten Creek RoadChapel Hill, USA.

${ }^{10}$ To whom correspondence should be addressed. (e-mail: joseph.w.polli@gsk.com)
AAPS began its transformation almost four years ago with the agreement to develop a five-year strategic plan (2016 to 2020), starting with a workshop at the 2015 Leadership Retreat where input was collected from a broad representation of member groups. The strategic planning activity included an in-depth review of AAPS' governance processes, scientific format/ content for both of its annual meetings (the AAPS Annual Meeting and Exposition and the National Biotechnology Conference $[\mathrm{NBC}]$ ), its internal organizational structure of sections and focus groups along with their responsibilities, and association finances. A vision for the future included staying abreast of the rapid advancements in pharmaceutical sciences, embedding the use of electronic communication platforms, and connecting members to a truly global community of scientists. The effort yielded the AAPS mission to "advance the capacity of pharmaceutical scientists to develop products and therapies that improve global health." This mission is to be achieved by (1) advancing scientific discovery, exchange, and learning; (2) expanding professional and public outreach; (3) enhancing member services; and (4) fostering AAPS' global community.

Where have we, the membership, traveled during this journey to AAPS 2020? It started with more than one thousand pharmaceutical scientists sharing what was going well in the association, what needed improvement, and what were the future opportunities and challenges for the organization's members. The AAPS Leadership learned about several important aspects including the members' expectations to:

- $\quad$ Ensure increasing depth, quality, and rigor in AAPS scientific programs;

- Develop new web-based tools, online communities, and resources to create a more robust online platform for networking and collaborating with fellow members;

- $\quad$ Provide additional opportunities for members to engage with the Association through volunteer leadership, committees, etc.;

- $\quad$ Strengthen existing AAPS products and services to deliver additional value to members; 
- $\quad$ Expand existing international programs, services, and collaborations; and

- Increase collaboration between AAPS and other scientific associations and organizations.

One of AAPS Leadership's first activities in the transformation process was to review the governance framework, which encompasses the practice, policies, and rules used by the Board of Directors (previously called the Executive Council) to ensure accountability and transparency to its stakeholders, with a primary focus on serving the AAPS membership. The governance framework is central to the delegation of responsibilities, distribution of rights, and stewardship of finances. It covers all members from the Board, to members participating on committees and working groups, to the association staff. The review found gaps in the AAPS governance framework and operating procedures that created risks, both legal and operational. The AAPS Bylaws were not consistent with current laws and best practices for 501(c)(3) not-for-profit organizations and needed to be updated. Other areas that required modernization included section/focus group operations, awards, and financial support provided to membership subgroups by the association. The governance changes recently implemented through bylaw and policy updates will enhance the prosperity and viability of the organization, empower our volunteers, and allow AAPS Leadership to better manage risk.

Supporting pharmaceutical scientists and their efforts to develop products and therapies that improve global health is the heart of AAPS and the very reason why the association exists to serve its members and the public. Science underpins the contributions of our members, their professional development, and how their research breakthroughs impact the health of people around the world. Previous annual meetings were the main venues for AAPS members to share research, exchange ideas, and advance knowledge in the pharmaceutical sciences. Annual meetings served the association well for many years. However, over the past ten years, pharmaceutical modalities, technologies, and drug products have become more complex and diverse; industry continues to undergo organizational and structural changes; academia is increasingly involved in developing products in addition to basic research activities; regulations have evolved to ensure the safety and efficacy of new medicines; and the ability to communicate rapidly across the globe has been revolutionary.

AAPS members expressed their expectation that the best scientific programming should be delivered in ways that provide flexibility and value, and that balance time away from work/family. Feedback from members and meeting attendees suggested that the annual meeting events should be renewed from both the program selection-process perspective and the scientific-content perspective. This led to sunsetting the two annual meetings and the creation of AAPS PharmSci 360 as the association's flagship meeting event. AAPS PharmSci 360 is designed to combine the energy of the two former scientific conferences with the intimacy of small niche gatherings through track-based compartmentalization, and the inclusion of both chemical entities and biomolecular drugs.

AAPS PharmSci 360 offers five robust tracks covering key aspects of the pharmaceutical sciences that are important focal points to our members: Preclinical Development, Bioanalytics, Clinical Pharmacology, Manufacturing and Bioprocessing, and
Formulation and Quality (1). Each track has subtracks devoted to chemical entities and biomolecules. PharmSci 360 has the latest, most innovative research in the field, and it increases the exposure of the attendees to new science through dynamic interactions in the Solution Center with exhibitors who are experts in their scientific and technical areas, and through personalized careerdevelopment sessions. At PharmSci 360, one can either focus on a single scientific subtrack (e.g., large molecule bioanalytical topics) for the entire three-day meeting or choose to attend different sessions across the five tracks covering both small and large molecules. PharmSci 360 will be held from Sunday to Wednesday, will offer free time to balance ongoing work-life demands, and will have fun philanthropic and physical activity events. The scientific and professional development content is bookended with two networking receptions.

Other strategic changes included rolling out the AAPS Communities, implementing electronic posters, creating volunteer opportunities, and updating the awards program. Information technology and AAPS members' preferences for networking, interacting, and collaborating are rapidly advancing. A common theme in the collected feedback emphasized AAPS delivering an enhanced platform for all members with a common interest to interact, expand our international programs and services, and increase collaboration with sister associations and organizations. To meet these requests, AAPS has implemented an online community platform. Over the past 12 months, 41 sections and focus groups have transitioned to the community platform. Many communities are actively interacting, exchanging scientific knowledge, and collaborating on programming ideas. These interactions are self-initiated and led by scientists in the community on topics they want to discuss. Moreover, communities provide a robust platform for members to communicate in real time year-round. Starting in early 2019, members can form new communities covering science, careers, or advocacy issues.

Transforming the section and focus group structure to communities was a major decision that changed the feel and culture of AAPS. Sections and focus groups were central to the Annual Meeting and NBC for delivering programming topics, for leadership development, and were the "tribes" for our core volunteer members. Indeed, it has been a challenging transition from sections and focus groups to AAPS Communities. The decision to sunset sections and focus groups was partially driven by the need to remove the existing sense of silos and expand member engagement more broadly outside of the core group of volunteers who served sections/focus groups. Further, the decision to sunset sections and focus groups also serves to (1) simplify the association's structure; (2) address the funding imbalance across sections; (3) update the fragmented travel support policy for speakers and students; (4) manage the increasing number of awards; (5) enhance the scientific depth and diversity at AAPS meetings; (6) provide a more open process for program selection that harnesses the full scientific acumen of the AAPS membership; and (7) allow for expand calls for volunteers to serve in the association. Finally, the foregoing changes help members to focus their volunteer time on science-related activities, not those activities related to managing the organization's processes and overhead. 
AAPS member engagement is one of the main drivers behind the strategic plan and includes support for students, AAPS chapters and career scientists advancing in their current or new professional positions. One of the key areas where the changes have enhanced member engagement is in the number of volunteer opportunities and the process whereby members are selected for roles on various AAPS committees and working groups. There is an open call for volunteers that provides an opportunity for any member to apply for a position or committee. Applications are anonymized and reviewed by independent selection teams. Service is an important aspect of being a member. Our goal is to connect as many members as possible to volunteer roles that allow them to apply their scientific expertise and passion to enhance their personal development.

The Awards Committee was charged with aligning the awards program with the AAPS mission, goals, and membership needs to create value. Over the years, there has been a proliferation in the number of awards to over one hundred, a reduction in overall quality and quantity of nominations, and a lack in the transparency regarding the purpose of the awards, all at a cost of more than $\$ 250,000$ annually. Furthermore, the association continues to receive new award requests in an external environment where obtaining sponsorship can be challenging from a cost and legal perspective. The revised awards program, implemented in 2018, focuses on significant recognition of scientific contribution and innovation, leadership, and service (2). This reflects the association's mission and core values. There are currently eleven major AAPS awards that will be presented at PharmSci 360. The Awards Committee will continue to review the program and is open to adding additional awards provided they are aligned with the mission of AAPS.

The AAPS Leadership recognizes that changes have come with some disruption to the membership and the association staff. We acknowledge that we did not always clearly communicate the need and reason, timing, and/or impact of the changes in policies and procedures to the level we desired. Clearer communication on the move to electronic posters is needed. It creates new options for presenting data, including videos and spontaneous sessions all week long at the central poster kiosk, a simple mechanism to request an electronic copy of the poster, and the possibility for archiving this content for members to access in the future. The move to electronic posters was based on feedback from members looking for more flexibility in presentation formats, the ability to modify poster content rapidly, the desire to include video, improving the ability to share content, and to reduce the cost of preparation. However, the transition has come with unexpected consequences and challenges. Significant and diligent effort has been expended to address these shortcomings for PharmSci 360. We are confident that the 2018 experience will be positive for both the poster presenters and viewers.

With a vision toward AAPS 2020, the past few years have seen a significant shift in governance and methods to deliver scientific content to both PharmSci 360 attendees and to members at remote locations. Strategic planning has enabled the AAPS Leadership to streamline numerous processes, manage the association's finances, and address information technology demands. This effort is almost complete, allowing the association to prosper, focus on its mission, and enhance the capacity of its members. We acknowledge the countless hours that AAPS members, volunteers, leaders, and staff have provided to develop and implement these needed changes. It is the belief of the AAPS Leadership that these changes will ensure the longevity of AAPS and its future success. Whether you are a long-term AAPS member, a recently joined new member, or a first-time attendee, we look forward to seeing you at PharmSci 360 and sharing our vision for AAPS 2020 with you.

Statement of Copublication This article was simultaneously published in AAPS Open, The AAPS Journal, and AAPS PharmSciTech. https://doi.org/10.1186/s41120-018-0028-y https:// doi.org/10.1208/s12248-018-0273-y or https:/doi.org/10.1208/ s12249-018-1200-3 The American Association of Pharmaceutical Scientists (AAPS) holds copyright for this article.

\section{ACKNOWLEDGEMENTS}

The authors would like to thank all our members for their service to AAPS.

\section{AUTHORS' CONTRIBUTIONS}

All authors wrote or contributed to the writing of the commentary and approved the final manuscript.

\section{COMPLIANCE WITH ETHICAL STANDARDS}

Availability of Data and Material A statement for availability of data and material is not applicable for this study.

Competing Interests All authors are elected AAPS Board of Director Members. The authors declare that they have no competing interests.

\section{REFERENCES}

1. American Association of Pharmaceutical Scientists. Annual Meeting: PharmSci 360. https://www.aaps.org/pharmsci/annualmeeting. Accessed 24 Sept 2018.

2. American Association of Pharmaceutical Scientists. AAPS Awards Guide. https://higherlogicdownload.s3.amazonaws.com/ AAPS/bfc3a388-f31c-452f-88fd-941c2a445a10/UploadedImages/ 2018_AAPS_Awards_Guide_v1_Final_Updated.pdf. Accessed 24 Sept 2018. 